\title{
Bienveillance et exigence pour un enseignement inclusif
}

\author{
Jean-Pierre Garel \\ Chercheur associé - Université de Bordeaux - LACES EA 7437
}

Reçu le : 02/12/2019 | Accepté le : 11/05/2020 Résumé : On se demande ici dans quelle mesure la bienveillance et l'exigence des enseignants peuvent favoriser une
dynamique inclusive pour des élèves en situation de handicap, plus généralement pour celles et ceux qui ont des
besoins éducatifs particuliers (BEP), et comment elles s'articulent. Une fois précisé le sens des notions en jeu, la
méthodologie retenue pour identifier des catégories de manifestations de bienveillance et d'exigence est présentée. II
s'agit d'une analyse de contenu thématique des propos de professeurs d'éducation physique et sportive intervenant
dans des classes ordinaires qui accueillent des élèves à BEP. La bienveillance et l'exigence étant considérées comme
deux dimensions de l'activité professionnelle des enseignants, il est procédé à une analyse de leurs propos sous cet
angle, en référence aux travaux de Bucheton et Soulé (2009). Elle aboutit à distinguer huit types de manifestation.
Ce sont ensuite leurs conditions de possibilité qui sont envisagées, en termes d'attention à la diversité des élèves et
d'actualisation de principes éthiques, puis leurs relations. Il en ressort le constat d'une fréquente intrication entre
bienveillance et exigence. Au final, il apparaît que les deux attitudes étudiées ont pu contribuer à une dynamique
inclusive et que la complexité du travail enseignant invite à réfléchir sur la formation des professeurs.

Mots-clés : besoins éducatifs particuliers - bienveillance - exigence - handicap - école inclusive.

Depuis le rapport Refondons l'école de la République (Dulot et al. 2012), la bienveillance occupe une place de choix dans les discours sur l'éducation. Elle fait l'objet d'une injonction récurrente qui vient croiser l'injonction à œuvrer pour une école inclusive et suscite des débats. Sa présence insistante, notamment dans les textes officiels de l'Éducation nationale, soulève des critiques, jusqu'à lui attribuer une fonction « simplement incantatoire, opportunément mobilisée pour pallier les difficultés du système éducatif à moindre coût »(Marcel, 2018). Dans les analyses qui lui sont consacrées, elle est souvent reliée à l'exigence, en s'y conjuguant mais aussi en s'y opposant. Ainsi, des professionnels de l'école expriment leur crainte qu'elle entraîne « une dérive laxiste » et que « l'école bienveillante soit une sorte d'élixir pour panser les maux de l'école, en dissimulant parfois des apprentissages insuffisants »(Saillot, 2018). Quant à l'exigence, elle aussi est l'objet de controverses. Dans une certaine mesure inhérente à l'éducation, elle est susceptible de créer « un climat d'autorité, d'ordre, d'incitation à l'effort et de concurrence afin d'ob- 
tenir les meilleurs résultats possibles ». En ce sens, elle nuirait au bien-être de l'élève (Sarremejane, 2017).

Dans le cas des élèves auxquels on pense le plus souvent à propos d'école inclusive, c'est-à-dire celles et ceux qui sont en situation de handicap, les questions au sujet de la bienveillance et de l'exigence se posent avec acuité. En effet, ils ne correspondent sans doute pas à l'élève idéal. Or, l'écart de l'élève réel par rapport à l'élève idéal peut se traduire par une réduction des exigences à son égard (Becker, 1952). Par ailleurs, et plus fondamentalement, on a là affaire à une population qui, confrontant à la vulnérabilité de la condition humaine (Gardou, 2006), peut susciter une bienveillance qui interroge : dans quelle mesure ne s'oppose-t-elle pas à des ambitions pédagogiques et éducatives qui appellent des formes d'exigence? A contrario, l'intégration de ces élèves au sein de l'école ordinaire a trop souvent été conditionnée par leur adaptation aux exigences scolaires « normales », au détriment d'une adaptation à leurs singularités et au point de constituer « un aspect de processus plus larges de sélection et d'exclusion » (Armstrong, 2001, p. 93). Dans cette situation, l'exigence est pour ces jeunes une source potentielle de mal-être pouvant hypothéquer la réussite scolaire, le développement personnel et la participation sociale de nombre d'entre eux. Les questions relatives à la bienveillance et à l'exigence apparaissent finalement comme une source de « dilemme professionnel et éthique », ainsi que mentionné dans un éditorial, intitulé « Situation de handicap : bienveillance ou exigence? », d'une revue professionnelle des enseignants d'EPS (éducation physique et sportive) (Becker, 2015).

La bienveillance et l'exigence pouvant donc avoir des effets positifs et négatifs, on se demandera dans quelle mesure elles peuvent favoriser une dynamique inclusive pour des élèves en situation de handicap, plus généralement pour celles et ceux qui ont des BEP (besoins éducatifs particuliers), et comment elles s'articulent. Après avoir précisé le cadre conceptuel et méthodologique de notre réflexion, on identifiera les manifestations de bienveillance et d'exigence de la part de professeurs d'EPS enseignant en collège, avant de considérer leurs conditions de possibilité et d'éclairer leurs relations.

\section{Cadre conceptuel et méthodologique}

II s'agit là de préciser ce que l'on peut entendre par enseignement inclusif, bienveillance et exigence, puis de présenter la méthode retenue pour notre étude.

\section{1 Éclaircissements sémantiques}

L'enseignement inclusif peut être défini comme un enseignement, assuré au sein de l'école ordinaire, qui s'attache à la réussite scolaire de tous les élèves, à leur épanouissement personnel et à des interactions sociales positives. On retrouve dans cette définition des idées-clés contenues dans un rapport scientifique sur l'école inclusive pour les élèves en situation de handicap (Ebersold et al., 2016).

Quant à la bienveillance, c'est, selon l'Académie française, un « sentiment qui porte à vouloir du bien à autrui » et qui est souvent associé au bien-être (Lapeyronnie, 2014 ; Bergugnat et al., 2017; Jellab et Marsollier, 2018). Gwenola Reto résume le lien qui les unit : de «l'École bienveillante, [...] on attend désormais qu'elle soit pourvoyeuse de 
bien-être » (Reto, 2017), une notion qui peut faire référence à « la santé psychosociale » (Courty et Dupeyron, 2017) ou signifier une « disposition agréable du corps et de l'esprit » (Académie française). Dans ce cas, il peut être entendu comme circonscrit au moment présent, une limite qui est dépassée dans une définition qui a par ailleurs l'intérêt d'évoquer le contexte comme facteur de bien-être : «Le bien-être est un état qui révèle un sentiment positif et agréable, durable, engageant la totalité de la personnalité dans un contexte luimême favorable »(Sarremejane, 2017).

Le contexte favorable tient notamment au climat scolaire (Debarbieux, 2015), facteur de réussite scolaire (Cohen, 2006). Or, en France, ce climat laisse à désirer (Algan et al., 2018). Son lien avec la bienveillance est explicitement avancé : « Être bienveillant [...] c'est créer un climat de classe où l'on peut se tromper, prendre des initiatives, essayer, sans risques réels [...]. C'est en réalité " bien veiller sur "»(Zakhartchouk, 2017). On peut ajouter que «c'est bien veiller à », tout particulièrement à ce « qu'ils (les élèves) se développent" bien ", dans toutes leurs dimensions »(Reto, op. cit.). Ce qui suppose de s'attacher aux apprentissages. Leur contribution au sentiment de bien-être fait comprendre qu'il « ne peut être séparé du rapport au savoir, de la mobilisation sur les contenus enseignés » (Jellab, 2018, p. 33) et qu'il est multiforme, recouvrant « plusieurs dimensions allant du sentiment d'efficacité personnelle (Bandura, 1986) au plaisir éprouvé lorsque, progressivement, la maîtrise des savoirs forge une certaine réalisation de soi »(ibid.). Cette réalisation personnelle passe par des savoirs reliant les individus. Ils convoquent une «bienveillance culturelle»(Roelens, 2017, p. 98), c'est-à-dire le souci de «" bien veiller" à ce que soit possible la nécessaire appropriation d'une culture historiquement construite », afin que l'individu « ait la possibilité de s'inscrire dans une temporalité et dans un univers symbolique de culture, en les comprenant et en pouvant se les approprier de façon critique »(Roelens, ibid.).

L'attention aux savoirs appelle, de la part des enseignants, une bienveillance impliquant des « postures professionnelles favorables aux apprentissages des élèves, soucieuses de penser et travailler les obstacles susceptibles d'entraver la motivation scolaire » (Jellab, op. cit., p. 37). À l'opposé d'une bienveillance comprise comme une indulgence, d' « "une conception molle" de la bienveillance qui ne s'appuierait plus sur l'exigence, mais sur les nécessités de la paix sociale ou le manque d'ambition pour certains élèves » (Zakhartchouk, op. cit., p. 11), on en vient à promouvoir une forme active de la bienveillance, qui invite à agir au mieux pour l'élève (Marsollier, 2018, p. 60), «à garantir ce qui est bien pour son développement »(ibid.), et donc à travailler à sa réussite scolaire.

Alors que la bienveillance donne lieu à de nombreuses injonctions et publications, il n'en va pas de même pour l'exigence. Définie par l'Académie française comme « ce qui est requis, commandé par les circonstances», on peut dire, concernant l'école, qu'elle porte sur les attentes à l'égard des élèves, notamment en termes d'acquisition de connaissances et de compétences. Dans ce sens, on admettra que « l'exigence est celle de l'apprentissage. Ce qui compte, c'est ce que les élèves ont appris, et durablement appris »(Zakhartchouk, 2012).

Au terme de cette approche de la bienveillance et de l'exigence dans le domaine de l'école, on retiendra que l'exigence porte sur les apprentissages et que la bienveillance se caractérise par un souci du bien-être de l'élève, mais aussi, possiblement, de ses apprentissages, 
rompant ainsi avec l'indulgence et la passivité qui lui sont souvent attribuées. Si donc les contours sémantiques du concept d'exigence paraissent bien délimités, ceux de la bienveillance sont moins clairs dès lors qu'ils incluent, et partagent avec l'exigence, une attention aux apprentissages. Ce flou ne manquera pas de poser problème lorsqu'il s'agira de distinguer ce qui, dans le travail de l'enseignant, relève de l'une ou de l'autre.

\subsection{Méthode}

Pour identifier les manifestations de bienveillance et d'exigence, les données qualitatives fournies par les documents retenus font l'objet d'une analyse de contenu (Paillé et Mucchielli, 2003).

\subsubsection{Le choix des documents}

Les documents choisis fournissent des données secondaires, c'est-à-dire qu'ils n'ont pas été produits pour l'étude présente. Ils sont issus de travaux portant sur l'activité professionnelle d'enseignants d'EPS intervenant auprès d'élèves à BEP scolarisés en établissement ordinaire. Jugeant que la bienveillance et l'exigence sont deux dimensions de cette activité, on a procédé à l'analyse de ces documents sous cet angle. Leur choix a été dicté par une attention à la diversité de la population considérée, de manière à y trouver des élèves présentant un éventail de caractéristiques les inscrivant dans la catégorie des BEP, en l'occurrence une déficience physique, une déficience intellectuelle, une maladie somatique et une obésité. II ne s'agissait pas de comparer l'activité des enseignants en fonction du type d'élèves auxquels ils s'adressent, mais de prendre en compte une diversité de situations. En effet, on ne peut pas exclure que leur attitude diffère plus ou moins selon les cas.

La déficience physique et les problèmes de santé, en l'occurrence une paraplégie, une myopathie de Becker, un spina bifida, une cardiopathie, une comitialité et une gonalgie, sont abordés à travers deux documents qui sont la retranscription d'entretiens menés personnellement dans un collège auprès de deux professeurs. II est à souligner que les entretiens ont eu lieu après que nous avons été observateur non participant de plusieurs cours de ces professeurs, ce qui a donné matière à un ancrage de leurs paroles dans la réalité de leur travail. Par ailleurs, la parole d'élèves ayant suivi les cours des professeurs interviewés a été recueillie, ainsi que celle d'un parent d'élève.

Un troisième texte est constitué par le mémoire de master 2 Recherche de Justine Colin (2011) sur le travail d'un professeur d'EPS avec des élèves ayant des troubles cognitifs, scolarisés en Ulis (Unité localisée pour l'inclusion scolaire) et accueillis au sein d'une classe de sixième. Ce mémoire avait fait l'objet d'un article dans une revue professionnelle d'enseignants d'EPS (Léziart, 2015). La recherche qu'il présente a notamment l'intérêt d'être focalisée sur une pratique effective. En effet, les cours du professeur sont observés et filmés par Colin, puis les images lui sont présentées pour des entretiens d'autoconfrontation. On s'est attaché aux propos du professeur et aux commentaires de Colin sur son travail.

Deux autres textes sont les mémoires professionnels relatifs à des élèves en situation d'obésité, produits par Florence Gonnet et Christelle Siusko, professeures d'EPS, pour l'obtention du diplôme d'enseignant.e spécialisé.e. Ce type d'élèves, en nombre croissant, était intéressant à considérer, car ils présentent des caractéristiques ne manquant pas d'interpeller la bienveillance et l'exigence des enseignants, d'autant plus que la cause d'une 
obésité est parfois plus ou moins attribuée à un manque de volonté de l'individu concerné. Nous sommes donc allé au Centre de documentation de I'INSHEA ${ }^{1}$ pour consulter les mémoires relatifs à cette population et nous avons gardé les deux seuls dont les auteurs exposaient et analysaient leur expérience personnelle avec ce type d'élèves.

Les cinq documents retenus mettent en avant une posture réflexive des professeurs sur leur pratique, mais qui n'est pas centrée sur leur rapport à la bienveillance et à l'exigence. Cependant, il y est bien présent, avec un avantage : pas engagés à aborder directement ce sujet, les enseignants pouvaient éviter de tenir le discours convenu et désirable que peut appeler une demande d'expression sur des domaines traversés par des valeurs.

\subsubsection{Le traitement des données}

À partir d'une lecture répétée et attentive, le corpus est traité systématiquement par un recensement et une classification des éléments pouvant exprimer de la bienveillance et de l'exigence. La connaissance de travaux sur l'activité de l'enseignant oriente ce traitement. Sachant que la bienveillance et l'exigence se manifestent à travers des gestes professionnels, on s'est d'abord référé à leur distinction en cinq composantes, opérée par Bucheton et Soulé (2009). Quatre d'entre elles sont apparues pouvoir constituer une grille de lecture pertinente : les savoirs visés, ici les objectifs de l'enseignant; le pilotage de la leçon, qui a trait à son organisation; l'atmosphère, qui fait écho au climat scolaire, et l'étayage, entendu comme l'aide apportée à l'élève durant son activité. Cette approche déductive, consistant à appliquer un cadre d'analyse existant, a été complétée par une démarche inductive conduisant à des catégories qui ne sont pas un décalque de catégories prédéfinies.

Pour identifier les segments de texte susceptibles d'être regroupés sur la base de leur correspondance thématique et créer ainsi des catégories conceptuelles en rapport avec la recherche, on a dégagé quelques indicateurs lexicaux pouvant exprimer de la bienveillance et de l'exigence : capacité, pouvoir, encouragement, confiance, discussion, parole, exigence, travail, progrès, apprentissage (re)groupement, règle, compétition, autonomie, intégration, adaptation, disponibilité, valorisation. Listés ici sous forme de substantif, ces termes se déclinent parfois sous d'autres formes grammaticales, mais ils ne prennent sens que dans leur contexte discursif. On a donc privilégié des unités d'enregistrement constituées de phrases révélant une idée de bienveillance et d'exigence, sans pour autant contenir obligatoirement un des indicateurs lexicaux mentionnés. Elles amènent à distinguer huit thèmes. Deux d'entre eux concernent le climat scolaire et expriment de la bienveillance : les marques de confiance en l'élève et une attitude d'écoute et de dialogue. Pour les autres thèmes, il est plus difficile de leur attribuer un sens univoque, en termes de bienveillance ou d'exigence. On se demandera donc ultérieurement dans quelle mesure ils témoignent de l'une ou l'autre de ces attitudes. En premier lieu, il s'agit des objectifs, complétés par la mention « suffisamment ambitieux »; ensuite, de deux thèmes concernant l'organisation de la leçon : l'adaptation des situations d'enseignement/apprentissage et l'évaluation, qualifiée d'encourageante; enfin, trois thèmes qui relèvent de l'aide apportée à l'élève lors de son activité : l'ajustement de la distance à l'élève, des interventions différenciées, et la disponibilité envers tous les élèves.

1. Institut national supérieur de formation et de recherche pour l'éducation des jeunes handicapés et les enseignements adaptés. 
Le petit nombre de textes réunis, ainsi que l'absence de quantification des données, limitent les inférences et les conclusions que l'on peut tirer de leur analyse. On ne saurait identifier toutes les figures possibles de la bienveillance et de l'exigence à partir d'un échantillon réduit. En revanche, il fournit un point d'appui à l'analyse de conditions de la bienveillance et de l'exigence, et il permet de questionner l'intrication de ces deux attitudes.

\section{Manifestations de bienveillance et d'exigence}

\subsection{Des marques de confiance en l'élève}

C'est d'abord la confiance dans les marges de progrès de l'élève, ainsi que le formule Pascale : «C'est important d'avoir pris conscience que ces enfants-là (en situation de handicap - ndlr) pouvaient faire des tas de choses. »Cette confiance s'exprime par des encouragements, dont Colin note l'omniprésence dans les propos adressés par Olivier à Maxime, avec «l'objectif de réinvestir l'élève en montrant qu'il est capable de réussir »; par exemple: «T'y arrives, comme ça, c'est ce que tu as fait tout à l'heure. Allez! Viens mon grand, on réessaye tous les deux [...] Allez, on va y arriver! » Dès lors qu'elle est manifeste, cette confiance est susceptible d'accroître la confiance en soi de l'élève. Stéphanie, paraplégique, élève en quatrième : « Les profs, des fois ils nous encouragent, ils nous disent "mais si, tu vas y arriver", et on se rend compte qu'on y arrive. Ils ont confiance en nous. »

La confiance en l'élève porte aussi sur sa parole. Claude en témoigne, qui croit sans difficulté Antony, fatigable en raison de la myopathie de Becker dont il est porteur : « S'il va demander à se reposer, ça va être une demande justifiée, et elle est bien acceptée. »

\subsection{Une attitude d'écoute et de dialogue}

Elle se traduit notamment par un questionnement des élèves, individuellement ou collectivement, sur leur vécu en classe. Olivier : « II est important de faire un bilan de leçon avec les élèves de l'Ulis pour savoir comment s'est passé leur inclusion. »

L'écoute et le dialogue ont permis le traitement du problème de Vivien, revenu très amaigri au sein de sa classe après un séjour dans un établissement dédié à la prise en charge de l'obésité. Il informe Florence, sa professeure, qu'il ne pourra pas aller à la piscine. «Après discussion, note-t-elle, Vivien a fini par m'avouer qu'il avait peur des réactions des autres devant ses cicatrices. [...] En plus d'être gros, il avait peur d'être pris pour un monstre ». Elle lui a alors proposé d'expliquer à l'ensemble de ses camarades ce qu'il avait vécu, ainsi que la nécessité de cette chirurgie plastique. Finalement, les élèves ont bien réagi, et Vivien a pu participer au cycle de natation « en renvoyant l'image de quelqu'un de courageux qui avait su affronter les difficultés et qui avait su résister à la douleur physique ».

Être à l'écoute, c'est être ouvert à l'élève, attentif à ce qu'il ressent, à ce qui le mobilise, au plaisir qu'il peut éprouver, ou non. Christelle se dit satisfaite qu'une adaptation pédagogique ait, pour un élève obèse, «favorisé une plus grande motivation et du plaisir dans la pratique ». En effet, ajoute-t-elle, « mon objectif est que l'élève apprenne tout en prenant du plaisir ». Son ouverture apparaît également dans l'intérêt qu'elle porte à l'implication de l'élève dans le choix des adaptations le concernant, puisqu'elle entend le « rendre acteur de ses apprentissages en expliquant et en discutant des aménagements ». De même, 
Florence veut faire de l'élève un « acteur de son intégration et non plus seulement le spectateur passif ».

Ajoutons qu'une attitude ouverte incite l'élève à se confier : «Quand on a des problèmes, dit Stéphanie, on peut leur en parler. »

\subsection{Des objectifs d'apprentissage suffisamment ambitieux}

Claude insiste sur la place qu'elle accorde aux apprentissages de ses élèves en situation de handicap : «II faut (qu'ils) sentent que ce qu'on leur demande c'est du travail, avec une évaluation, qu'ils vont apprendre. »Pascale va dans le même sens : « Avoir la volonté de ne pas regarder ces enfants autrement [...], et d'avoir quand même des exigences avec eux. »

Cela dit, les réussites atteignables par un élève, du moins à court terme, peuvent être modestes. Pour Kevin, qui apprécie le tir à l'arc mais est très maladroit, Claude a prévu des objectifs et un travail à sa mesure : « II y avait tout un travail pour prendre la flèche, mettre la corde dans l'encoche. Ça a demandé presque deux séances pour qu'il soit autonome à ce niveau-là. II a des essais supplémentaires. On essaie de valoriser ses progrès. »

\subsection{L'adaptation des situations d'enseignement/apprentissage}

Le groupement des élèves en est un aspect. Par exemple, avec une adolescente obèse, Florence privilégie parfois des regroupements affinitaires. En effet, elle a remarqué qu'elle n'était pas toujours bien acceptée, car, avance-t-elle, « les difficultés de l'élève obèse ne déclenchent guère les réactions positives souvent suscitées par d'autres pathologies ». Pour la pratique de la boxe française, Christelle a également regroupé les élèves sur un critère d'affinité afin d'établir un climat de confiance, « de mieux appréhender la peur du contact ». Quant à Olivier, il veille à ce que chacun des élèves de l'Ulis soit avec des partenaires qui le prennent convenablement en compte lors d'une activité sur les arts du cirque.

Les enseignants adaptent aussi l'activité à partir de variables didactiques, par exemple en jouant sur l'espace d'action et les règles. Ainsi, Antony joue au badminton sur un terrain dont les dimensions réduites ne l'obligent pas à courir, contrairement à son adversaire, et les règles du basket-ball sont adaptées pour Marie-Laure, en fauteuil roulant. Enfin, « pour que la pratique physique reste motivante, analyse Florence, il est important de regarder la compétition avec un œil critique :" Qui a couru le plus vite, qui a sauté le plus loin?" L'adolescent obèse part forcément perdant ».

\subsection{L'ajustement de la distance à l'élève}

La distance à l'élève est modulée selon les cas. Avec Myriam, note Colin, Olivier est juste à côté d'elle pour lui parler et son regard est tourné vers elle, car, explique-t-il, « Myriam a besoin de se sentir en confiance ». II va même jusqu'à toucher les élèves pour montrer qu'il est proche d'eux. De façon générale, cependant, il cherche à maintenir une distance optimale pour éviter une surprotection : «II faut qu'ils se coupent un peu de moi et qu'ils prennent un petit peu de l'autonomie [...]. Si je reste tout le temps avec eux [...], ils ne sont pas vraiment dans les conditions d'intégration. » De son côté, Claude avance, 
à propos de ses élèves en situation de handicap, qu'il est « important de travailler en autonomie, de se prendre en charge ». Pascale, quant à elle, est consciente que, pour certains de ces élèves, il est parfois « difficile d'être au milieu d'un groupe dynamique ». Toutefois, ajoute-t-elle, « je crois que c'est important qu'ils soient dans une ambiance où il y a du monde autour d'eux qui les prend en considération sans s'arrêter systématiquement sur leur handicap ».

\subsection{Des interventions différenciées}

L'intervention, c'est-à-dire le fait d'engager une action auprès de quelqu'un pour résoudre un problème, s'avère différente selon les élèves. Colin relève qu'Olivier peut s'adresser sèchement à François : "Tu veux pas le faire, tu t'assois. Tu veux pas travailler, on arrête. C'est pas grave mais on arrête. » Alors que, remarque Olivier, « avec Max, je suis pas du tout dans ce dialogue-là. Je vais le rechercher sans arrêt ». À propos d'une autre élève, Colin observe : «Olivier s'adapte à Camille pour ne pas la brusquer, tenter de la réinvestir de manière délicate. »Olivier s'en explique : «II faut prendre des pincettes pour réinvestir Camille, et non imposer. »Et il justifie son attitude à l'égard de François : «L'objectif c'est quand même qu'il réintègre une Segpa [...], donc, en fait, le niveau d'exigence est pas tout à fait le même. »

\subsection{La disponibilité envers tous les élèves}

Colin note qu'Olivier conçoit son enseignement « avec l'idée de répondre aux besoins de chaque élève, et non d'apporter un soutien spécifique à des élèves stigmatisés comme handicapés », et elle souligne un dilemme qui est au cœur de l'activité du professeur : « Être disponible pour les élèves de l'Ulis tout en assurant la gestion d'une classe de trente élèves. »Même s'il sait que «les élèves de l'Ulis sentent qu'ils sont noyés un petit peu dans le groupe », Olivier confirme que « l'enseignant ne peut pas s'occuper exclusivement des élèves de l'Ulis ». De son côté, Pascale évoque sa prise en compte de tous les élèves : « II y a des enfants qui sont très mal dans leur peau. On les regarde autrement. (On arrive) à accepter de différencier davantage, même chez les valides. »

\subsection{Une évaluation encourageante}

Dans sa dimension formative, l'évaluation peut être instituée, ayant lieu au terme d'une séquence d'enseignement/apprentissage. Florence veut en faire « un outil de construction d'une meilleure image de soi ». À cet effet, avec un élève obèse, elle propose de faire régulièrement le bilan de ce qu'il a réussi à effectuer. Dans ces conditions, avance-telle, il ne se voit plus comme « celui qui ne sait rien faire mais celui qui est capable de progresser, et cela change tout! ». L'évaluation formative peut aussi être informelle, s'exerçant à n'importe quel moment de l'activité de l'élève, avec notamment l'objectif de le mettre en valeur pour qu'il ne se décourage pas. Elle est illustrée par Olivier à propos de Lucie, qui réalise une figure de jonglage différente de celle qui est prescrite : « $A$ chaque fois j'essaye de trouver un point positif; donc comme elle ne réussit pas mais comme elle fait l'autre mouvement, plutôt la valoriser sur le fait qu'elle réussit à le faire et que maintenant il faut qu'elle passe à l'autre. » Toujours en jonglage, il valorise Maxime, qui n'a pas réussi à réaliser ce qui était demandé, aux yeux des autres élèves : «T'as inventé un mouvement! II est bien!» 


\section{Conditions de la bienveillance et de l'exigence}

Deux types de conditions sont envisagées : l'attention à la diversité des élèves, avancée comme un principe directeur d'une démarche inclusive (Ebersold et al., op. cit.), et l'actualisation de principes éthiques porteurs de valeurs orientant la conduite de l'enseignant.

\subsection{L'attention à la diversité des élèves}

La diversité des élèves est à la fois interindividuelle et intra-individuelle, c'est-à-dire qu'ils différent entre eux et que chacun présente une grande variété de capacités, de compétences, de caractéristiques personnelles, telles que ses performances peuvent varier selon les situations auxquelles il est confronté. Cette diversité, ou variabilité, intra-individuelle (Ribaupierre, 2015 ; Fayol, 2015) invite à connaître suffisamment l'élève pour identifier les processus de son activité, les obstacles qu'il rencontre et les ressources dont il dispose. Les manifestations de bienveillance et d'exigence qui ont été présentées témoignent de l'attention des professeurs à connaître au mieux chacun et chacune, sans pour autant, notamment à travers une attitude d'écoute et de dialogue, se prendre pour le « psychologue de service », au risque de verser dans une « inquisition bienveillante » (Meirieu, en ligne) et l'illusion d'une connaissance totalisante.

L'attention à la diversité des élèves peut être abordée sous l'angle de leurs besoins. Commençons par regarder la place de trois besoins psychologiques fondamentaux, décrits par Ryan et Deci (2002, cités par Dupont et al., 2009), dans les manifestations de bienveillance/exigence qui ont été identifiées. Le besoin d'appartenance sociale, compris comme «besoin de se sentir appartenir à un groupe et d'être reconnu par celui-ci » (Ryan et Deci, op. cit., p. 17), est pris en compte lorsque l'enseignant veille à ce qu'un élève soit bien accepté par ses camarades, par exemple en constituant un groupe affinitaire ou en organisant une discussion collective qui permette à un adolescent d'expliquer l'épreuve qu'il a traversée. Pour sa part, le besoin d'autonomie est une préoccupation des professeurs qui est notable dans l'ajustement de leur distance à des élèves dont la déficience pourrait inciter à les surprotéger. Enfin, le besoin de compétence, c'est-à-dire de s'estimer compétent, appelle des mises en œuvre didactiques et pédagogiques adaptées, différenciées autant que de besoin (Garel, 2016). Elles mobilisent notamment, chez le professeur, une bonne connaissance de sa discipline et la capacité à identifier les entraves à la réussite des élèves, ainsi que les points d'appui sur lesquels leurs progrès pourront se construire. Ajoutons que satisfaire au besoin de compétence de l'élève implique que sa réussite soit reconnue par lui et par d'autres, car se reconnaître et se voire reconnu une capacité d'agir répond à un besoin essentiel, puisque la reconnaissance « constitue la validité nécessaire de la construction de soi »(Gardou, 1998, p. 99). De ce point de vue, une meilleure évaluation des élèves, « pour peu qu'elle soit compréhensive, régulière et davantage formative »(Jellab, op. cit., p. 43), est une condition fondamentale d'une école bienveillante. II ne s'agit pas de leurrer l'élève sur ce qu'il arrive à réaliser, mais de signaler les aspects positifs.

Ces types de besoins, comme d'autres, se déclinent de façon singulière selon les élèves, appelant des réponses adaptées à chaque cas particulier. S'agissant des élèves en situation de handicap, il convient donc de se détacher d'un préjugé induit par la catégorisation médicale. II consiste à leur attribuer des caractéristiques et des besoins identiques lors- 
qu'ils relèvent d'un même groupe nosographique - «les infirmes moteurs cérébraux 》, «les aveugles », etc. - alors que les individus faisant l'objet d'une même désignation ne constituent pas un ensemble homogène.

Il faut aussi souligner que les besoins dépendent des situations dans lesquelles se trouve l'élève. L'approche situationnelle du handicap, promue par l'OMS (Organisation mondiale de la santé) à travers la CIF (Classification internationale du fonctionnement, du handicap et de la santé - 2001), a mis en avant le rôle déterminant de l'environnement concernant les possibilités d'une personne. Par conséquent, dans la perspective d'une accessibilité pédagogique optimale des savoirs visés, identifier les besoins d'un élève suppose de considérer les obstacles liés à la situation dans laquelle il se trouve : de quelle nature sont-ils? Quelles adaptations de la tâche proposée sont à prévoir pour les surmonter? Quelles sont les médiations à mettre en œuvre?

Par ailleurs, l'attention aux besoins particuliers ne doit pas porter uniquement sur ceux qui tiennent à une insuffisance de l'élève, et l'enfermer ainsi dans des représentations péjoratives qui le constituent comme un être à part. Ce n'est parce que des enfants et des adolescents présentent une déficience ou une maladie importante qu'ils ne partagent pas des besoins et des désirs avec leurs camarades typiques, qu'ils ne recherchent pas le plaisir du jeu et du sport (de Barbot, 1987) et qu'ils doivent être tenus à l'écart d'activités dont on pense qu'elles ne sont pas faites pour eux. II s'agit également d'être ouvert aux capacités individuelles pour y étayer des progrès. Cette attitude accueillante a autorisé des élèves à inventer, avec succès, des techniques improbables, telle la saisie d'une raquette de tennis de table avec les pieds ou la bouche.

La diversité des élèves tenant aussi à leur rapport au savoir, le sens qu'ils attribuent à leur activité mérite une attention. Ce sens peut tenir au sentiment de compétence, à l'autonomie ou à l'appartenance sociale qu'elle leur apporte, mais pas uniquement. II faut aussi que cette activité puisse, autant que possible, répondre à des problèmes qu'ils rencontrent et qu'ils se posent, et, fondamentalement, qu'elle suscite en eux des émotions mobilisatrices qui sont souvent propres à l'EPS. Certes, comme les autres disciplines, I'EPS implique des apprentissages, mais dans des domaines où des plaisirs singuliers le disputent à l'effort. Enraciné dans le jeu, le sport est source de sensations corporelles et d'émotions particulières (Jeu, 1977), de même que des pratiques physiques qui ne sont pas sportives, au sens strict, telles celles qui sont de nature artistique ou qui font partie du patrimoine ludique enfantin.

\subsection{L'actualisation de principes éthiques}

C'est essentiellement dans le cadre des écrits sur la bienveillance que l'on trouve des études sur les principes éthiques susceptibles d'orienter la conduite de l'enseignant. Toutefois, postulant que la bienveillance et l'exigence ne se distinguent pas toujours aisément, on avancera que les mêmes principes peuvent valoir plus ou moins pour l'une et l'autre de ces dispositions, ainsi qu'on le verra pour chacun d'eux. Certains d'entre eux relèvent d'une approche déontologique, selon laquelle un devoir s'impose en toutes circonstances, en référence à la morale kantienne. D'autres sont de l'ordre de la vertu. « Capacité à apprécier et à faire » (Prairat, 2013, p. 175), la vertu « n'est pas seulement une disposition à bien agir, elle est aussi une disposition à bien ressentir » (ibid, p. 176), qui 
conduit l'enseignant à se fonder sur son jugement pour décider de son attitude ou de l'action à entreprendre dans une situation donnée, dans l'intérêt de l'élève.

\subsubsection{Un regard positif sur l'élève}

Le « rapport positif à la diversité des profils scolaires » est une caractéristique forte de l'éducation inclusive (Ebersold et al., op. cit., p. 17). II s'exprime notamment par un regard positif sur l'élève, un impératif catégorique qui fait écho au devoir de confiance (Prairat, 2012) envers ses capacités. Permettant de formuler des objectifs suffisamment ambitieux à son égard, il repose sur le pari de l'éducabilité. En fait, la confiance ne relève pas d'un pari, au sens d'un espoir que la chance sourie, mais du constat de réussites qui tiennent moins au hasard qu'à un enseignement bien adapté. La confiance manifestée à l'élève alimente sa confiance en soi, facteur d'engagement dans une activité.

Porter un regard positif sur l'élève ne signifie pas que l'enseignant accueille favorablement tout ce qu'il fait. Ainsi, la bienveillance d'Olivier ne l'empêche pas de marquer son désaccord avec l'attitude de François. De fait, à verser dans l'indulgence systématique et l'évitement de la confrontation, on « maintient (l'élève) dans l'illusion narcissique de la toute puissance et prépare des lendemains qui déchantent »(Bernardin, 2013).

Le regard positif sur l'élève s'inscrit clairement dans le champ de la bienveillance, mais, par les limites qu'il s'impose, il n'est pas totalement exempt d'exigence.

\subsubsection{La sollicitude}

Vertu du maître de l'école inclusive (Prairat, 2013), la sollicitude est présentée par Fabienne Brugère comme « la capacité de se soucier des autres et la conduite particulière qui consiste à se préoccuper d'autres identifiés par un besoin ou une vulnérabilité trop grande » (Brugère, 2011, p. 25), ou encore comme « une certaine relation aux autres sur le mode de la protection ou de la préoccupation »(Brugère, 2008, p. 13). Avoir conscience d'une vulnérabilité est important, mais sa mise en avant risque de dessiner l'image d'un élève fragile, faible, à protéger, dont il faut prendre soin plutôt que de le confronter à des obstacles dont le franchissement l'engagerait dans une voie de progrès. On a vu, en EPS, des élèves en situation de handicap « bénéficier», de la part de professeurs mus par une compassion envahissante, d'une aide si conséquente qu'ils ne pouvaient pas s'attribuer leur réussite, devenir sujet de leurs actes et donc s'émanciper d'une dépendance à un enseignant omniprésent (Garel, 2018 ; Marcel, 2018). Pour reprendre la distinction opérée par Heidegger entre deux types de sollicitude (Heidegger, 1986), il s'agit donc de privilégier une sollicitude émancipatrice, à l'opposé d'une sollicitude qui assujettit la personne en se substituant à elle.

Entendue comme «attention à celui qui est en difficulté »(Prairat, 2013, p. 183), la sollicitude se retrouve dans les médiations mises en œuvre par l'enseignant pour accompagner l'élève dans son activité, telles des marques de confiance, l'ajustement de sa distance, sa disponibilité, et une attitude d'écoute et de dialogue qui a conduit, par exemple, un professeur à se préoccuper des raisons pour lesquelles un élève refusait d'aller à la piscine. 
La sollicitude peut être considérée comme une vertu exemplaire de la bienveillance. Cependant, pouvant être mobilisée dans la recherche d'adaptations favorables aux progrès, elle n'est pas dénuée d'exigence.

\subsubsection{Le tact}

Vertu relationnelle par excellence (Prairat, ibid., p. 181), le tact est à comprendre comme la « conscience aigüe de ce qui mérite d'être dit ou d'être fait et de la manière dont il faut le dire ou le faire (dans) la situation particulière que l'on est en train de vivre » (Prairat, 2017, p. 13). Il est particulièrement mobilisé par l'enseignant pour ajuster sa distance à l'élève, différencier ses interventions et moduler ses exigences et son soutien dans les situations complexes, sources de dilemme, ainsi que le remarque Colin à propos d'Olivier, souvent face à un choix difficile lors de son intervention auprès des élèves puisqu'il lui « est nécessaire de trouver un compromis entre exigence du cadre scolaire et possibilités de l'élève au moment ». Un dilemme, présenté par Marsollier, interroge tout particulièrement la distinction entre bienveillance et exigence. Au nom de l'éthique en jeu dans la bienveillance, avance-t-il, l'enseignant peut être conduit, dans l'intérêt de l'élève, « à "sacrifier" les enjeux immédiats et à imposer une exigence fortement contraignante » (Marsollier, op. cit., p. 76).

Un autre dilemme que rencontrent parfois des enseignants est illustré par l'exemple de Christelle, demandant à l'un de ses élèves, obèse, pourquoi il refuse les adaptations proposées. Sa réponse : « Je ne veux pas être différent des autres. »Dans ces conditions, soit le professeur ne différencie pas son enseignement, l'élève risquant alors d'être en échec et de mal le vivre, soit l'adaptation est vécue comme une stigmatisation.

La dimension relationnelle du tact le place du côté de la bienveillance, mais, pour cette vertu comme pour d'autres, le souci des apprentissages le dispute à celui du bien-être.

\subsubsection{Le sens de la justice}

«Vertu majeure de l'enseignant »(Prairat, 2017, p. 181), le sens de la justice porte à conjuguer l'égalité des droits et l'équité de traitement des élèves. Il invite donc à veiller à ce que chacun soit confronté à des contenus d'enseignement et à des situations suffisamment ambitieux et adaptés pour lui permettre de progresser. De même que le tact, le sens de la justice est source de dilemmes. Dans quelle mesure, par exemple, accorder à un élève avec une déficience un droit particulier, pour participer à une activité collective, qui ne lui donne pas trop de «facilités » par rapport à ses capacités, et donc ne l'assigne pas à sa différence, et qui soit bien accepté par ses camarades?

En s'appuyant sur des compétences didactiques et pédagogiques, le sens de la justice conditionne l'adaptation des situations d'enseignement/apprentissage à tous les élèves. II conduit donc l'enseignant à distribuer son attention entre tous et toutes, faisant ainsi face à une exigence complexe de son métier : «Saisir à la fois les individualités dans leur singularité en les conjuguant au pluriel »(Reto, op. cit.). Les dilemmes qu'il provoque résultent d'une bienveillance empreinte de la vigilance à ce que tous et toutes soient en situation de réussite. 


\section{Les relations entre bienveillance et exigence}

Le regard porté sur les conditions de la bienveillance et de l'exigence a montré la complexité des situations dans lesquelles toutes deux sont à l'œuvre ainsi que la porosité de leurs relations. Ainsi, les analyses portant sur l'actualisation de principes éthiques se sont conclues, pour chacune d'elles, par le constat d'une infiltration de la bienveillance par une certaine forme d'exigence. II s'agit maintenant de s'appuyer sur les manifestations de bienveillance et d'exigence pour approfondir le type de relations qu'elles entretiennent et souligner le caractère complexe d'un enseignement qui les mobilisent.

\subsection{L'opposition}

À propos de ses élèves avec une déficience motrice, une professeure témoigne : «Ma principale difficulté a été d'avoir tendance à être trop tolérante [...]. J'ai été plus indulgente $[. .$.$] . On a tendance à faire passer tout ce qui est affectif un petit peu au-devant$ du travail, du niveau, des exigences. » On peut voir dans la tolérance une expression de la bienveillance, mais ici sur un mode passif qui pose question. II faut dire que la « tolérance à la différence » fait écho au respect des différences, principe souvent avancé dans les réflexions éthiques sur le rapport à l'altérité, mais qui est discutable dès lors que le respect laisse entendre une limite de l'action à l'égard de l'objet respecté. Cette retenue peut alors prendre la forme d'une tolérance qui fait de la différence « une donnée objectivée, naturalisée, insurmontable, à laquelle il convient de s'adapter »(Joshua, 1999, p. 21). Auquel cas la bienveillance empêche l'exigence.

\subsection{L'intrication}

Hormis les exemples de confiance en l'élève et d'attitude d'écoute et de dialogue, qui signent un vif souci de bien-être et penchent nettement du côté de la bienveillance, les manifestations de bienveillance et d'exigence que l'on a présentées mettent en jeu l'une et l'autre de ces dispositions, à travers l'attention au bien-être en même temps qu'aux apprentissages. Ainsi, l'ajustement de la distance à l'élève concilie l'objectif d'autonomie et la prise en compte d'un éventuel besoin de proximité entre le professeur et l'élève. Pour leur part, les interventions de l'enseignant visent à créer les conditions optimales d'un progrès de l'élève, et donc à établir avec lui des relations positives : si Olivier « prend des pincettes » avec Camille, c'est parce que c'est, selon lui, le meilleur moyen de la (re)mobiliser pour un apprentissage, faute de quoi il n'obtiendrait rien d'elle. Avec la définition d'objectifs suffisamment ambitieux, on a l'expression d'une exigence optimale, mesurée à l'aulne des possibilités de l'élève. En atteste l'exemple de Kevin, puisque la prise en compte de sa grande maladresse conduit son professeur à formuler des objectifs loin des standards. Quant à l'adaptation des situations d'enseignement/apprentissage, elle implique de veiller à l'adéquation des obstacles rencontrés par les élèves aux capacités et aux mobiles de chacun et de chacune, pour que leur franchissement constitue un progrès significatif. Pour ce qui est de l'évaluation encourageante, évaluer les apprentissages s'accompagne d'une attention à mettre en lumière les résultats positifs. Enfin, la disponibilité envers tous les élèves, et pas seulement en direction de ceux désignés comme étant en situation de handicap ou à besoins particuliers, traduit l'exigence de bien veiller aux singularités de tous et de toutes, y compris en termes d'apprentissage. 


\subsection{Entre bienveillance et exigence, un écart, plutôt qu'une différence}

Au premier abord, la bienveillance peut sembler distincte de l'exigence. D'ailleurs, une expression telle que «la bienveillance permet l'exigence »(Zakhartchouk, op. cit.), qui souligne le lien qui les unit, met en scène deux mots, donc, a priori, deux entités différentes. Pourtant, qualifier de différence ce qui sépare ces deux notions est discutable. Même si la bienveillance dirige l'attention vers le bien-être et le climat scolaire, tandis que l'exigence est tournée vers les apprentissages, il existe entre elles un écart plutôt qu'une différence. Écart et différence «marquent une séparation, mais la différence sous l'angle de la distinction, l'écart sous celui de la distance »(Jullien, 2019, p. 57). Alors que la différence « sert à identifier et classer [...], l'écart, en ouvrant une distance, engage, non pas une identification, mais une exploration. Jusqu'où va l'écart? ». Dans cette perspective, la bienveillance et l'exigence sont ainsi maintenues en regard et en tension, suscitant une réflexion sur ce qui les rapproche et les éloigne. La tension qui les anime se manifeste par une oscillation entre les deux pôles qu'elles constituent, plus que par le maintien d'un point d'équilibre à égale distance de chacun d'eux. À l'égard d'un même élève, l'enseignant peut poser des exigences élevées sur certains aspects, à certains moments, et, dans d'autres circonstances, se montrer davantage attentif à son bien-être dans l'ici et maintenant.

Maintenir en regard et en tension deux éléments habituellement séparés relève d'une conception épistémologique et philosophique qui, chamboulant les étiquettes et traversant les frontières (Morin, 1990), amène à considérer les personnes « handicapées », par rapport aux autres, comme des personnes dont les singularités signent moins des différences de nature que de degré. Ce qui, dans une perspective inclusive, invite à une double exigence : veiller à prendre en compte le singulier de chaque individu et à construire du commun (Garel, 2018).

\section{Conclusion}

Ayant pris des distances avec une acception courante de la bienveillance qui lui donne pour synonymes la bonté, l'indulgence et la complaisance ${ }^{2}$, et qui, mettant excessivement en avant la vulnérabilité, risque de se traduire par une attitude compassionnelle envers l'élève au point de substituer le soin à la justice (Michaud, 2016), on est arrivé à souligner sa proximité avec l'exigence. De fait, l'examen de leurs manifestations et de leurs conditions a fait souvent apparaître leur intrication. Être bienveillant, au sens de veiller au bien de l'élève, conduit à être suffisamment exigeant pour favoriser ses apprentissages, le développement de ses potentialités, son émancipation et sa participation sociale. D'un autre côté, la pertinence de l'exigence passe par une forme de bienveillance, en l'occurrence la nécessité de bien veiller à ce que les contenus d'enseignement, les tâches et l'accompagnement de l'élève soient adaptés de manière à constituer un environnement capacitant (Falzon, 2013), pourvoyeur d'un pouvoir d'agir infiltré par un désir et un plaisir d'agir.

En conjuguant bienveillance et exigence, les enseignants dont les propos ont été rapportés ont pu favoriser pour leurs élèves une dynamique inclusive dans la mesure où leur action a favorisé des apprentissages, un épanouissement personnel et des interactions sociales positives. La mère de Julie évoque l'évolution de sa fille par rapport à l'EPS : «Elle se

\footnotetext{
2. Selon le dictionnaire électronique des synonymes du Crisco (Centre de recherches interlangues sur la signification en contexte), ce sont, par ordre décroissant, les synonymes les plus fréquents de la bienveillance. 
sent comme les autres. C'est très important, surtout à cet âge-là : elle peut faire du sport, elle n'est pas rejetée, pas handicapée. Les autres années, elle ne se sentait pas bien dans sa peau d'être exclue. » Julie souligne l'importance de réussites qui contribuent à son bienêtre : «Je m'amuse bien [...]. Je n'aimais pas le sport parce que je ne réussissais pas bien. »Quant à Antony, dispensé d'EPS les années précédentes, il fait comprendre l'intérêt des interactions sociales dans un contexte adapté, puisque sa motivation première pour l'EPS, malgré les contraintes de sa myopathie, est le désir de « retrouver les copains ».

II ne s'agit pas d'attribuer uniquement les réussites qu'expriment ces témoignages à une attitude bienveillante et exigeante. Disons qu'elle y contribue, en satisfaisant aux conditions envisagées, nécessaires mais pas suffisantes. En effet, cette attitude des professeurs, fondée notamment sur l'attention à la diversité des élèves et sur l'actualisation de principes éthiques, doit s'appuyer sur de solides compétences professionnelles. De ce point de vue, il convient de mettre en avant l'importance de la formation des enseignants pour qu'ils soient mieux outillés face à la complexité de leur métier. On a montré plusieurs aspects de cette complexité, en particulier des tensions entre des principes et des orientations de l'enseignement convoqués par la problématique de la bienveillance et de l'exigence : bien-être et effort d'apprendre, égalité des droits et équité de traitement, attention à chacun et à tous et toutes, sollicitude et autonomisation, regard positif sur l'élève sans le leurrer, différenciation sans exclusion. Confronté à des situations complexes et singulières, l'enseignant ne peut se satisfaire des prescriptions de «bonnes pratiques » relevant d'un prêt-à-porter mal ajusté. D'où l'intérêt de dispositifs de formation comportant l'analyse de situations professionnelles porteuses de problèmes et d'incidents critiques (Pastré et al., 2006). En développant la capacité à observer et à analyser l'activité d'élèves et de leurs professeurs, ils contribuent à façonner des enseignants réflexifs et critiques (Perrenoud, 1999), concepteurs de leur pratique.

\section{Références bibliographiques}

Armstrong, F. (2001). Intégration ou inclusion? L'évolution de l'éducation spéciale en Angleterre. Une étude de cas. Revue française de pédagogie, 134, 87-96.

de Barbot, F. (1987). Enfant handicapé moteur : enfant malade? In P. Ferrari et R. Lazarovici (Eds.). L'enfant malade et son corps, Lieux de l'enfance (pp. 231-257). Toulouse : Privat

Becker, H. S. (1952). Social-Class Variations in the Teacher-Pupil Relationship. Journal of Educational Sociology, 25(8), 451-465.

Bergugnat, L., Dugas, É. et Malet, R. (Eds.) (2017). Le bien-être à l'école : un processus de production du bien-être? Tome 1 et 2 , Recherches \& Éducations, Revue de la société Binet-Simon.

Brugère, F. (2008). Le sexe de la sollicitude. Paris : Seuil.

Brugère, F. (2011). L'éthique du care. Paris : PUF, coll. Que sais-je?

Cohen, J. (2006). Social, emotional, ethical and academic education : Creating a climate for learning, participation in democracy and well-being. Harvard Educational Review, 16(2), 201-237.

Courty, B., Dupeyron, J.-F. (2017). Le bien-être des écoliers. Bordeaux : Presses Universitaires de Bordeaux.

Debarbieux, É. (2015). Définitions, effets, et politiques publiques. Éducations \& formations, 88-89, 11-27.

Falzon, P. (2013). Ergonomie constructive. Paris : PUF.

Gardou, C. (1998). La personne handicapée : d'objet à sujet, de l'intention à l'acte. La Nouvelle revue de l'AIS, 4, 97-109. 
Gardou, C. (2006). Fragments sur le handicap et la vulnérabilité. Pour une révolution de la pensée et de l'action. Toulouse : Eres.

Garel, J.P. (2016). Différencier pour inclure en EPS. Les Cahiers pédagogiques, 526, 47-48.

Garel, J.-P. (2018). Handicap : les pièges de la compassion. Les Cahiers pédagogiques, 542, 50-51.

Garel, J.-P. (2018). Élèves à BEP (Besoins éducatifs particuliers) en EPS à l'école ordinaire : intérêt et limites des types de groupement. La dialectique du singulier et du commun. La nouvelle revue-Éducation et société inclusives, 81, 81-97.

Heidegger, M. (1986). Être et Temps (F. Vezin, Trad.). Paris : Gallimard, coll. Bibliothèque de philosophie. (Édition originale : 1927)

Jellab, A. et Marsollier, C. (dir.) (2018). Bienveillance et bien-être à l'école, plaidoyer pour une école humaine et exigeante. Marseille : Berger-Levrault.

Jellab, A. (2018). La bienveillance, un nouveau care en éducation ? Plaidoyer critique et constructif pour une notion pédagogiquement vive. In A. Jellab et C. Marsollier, Bienveillance et bien-être à l'école, plaidoyer pour une école humaine et exigeante (pp. 19-21). Marseille : Berger-Levrault.

Jeu, B. (1977) Le sport, l'émotion, l'espace. Paris : Vigot.

Joshua, S. (1999). L'école entre crise et refondation. Paris : La Dispute.

Jullien, F. (2019). De l'écart à l'inouï. Paris : L'Herne.

Léziart Y. (2015). Recherche et handicap. Entretien avec Justine Colin. Contrepied, 12, 41-42.

Marsollier, C. (2018). De la nécessité d'accorder toute sa valeur éthique à la bienveillance active. In A. Jellab et C. Marsollier, Bienveillance et bien-être à l'école, plaidoyer pour une école humaine et exigeante (pp. 57-79). Marseille : Berger-Levrault.

Michaud, Y. (2016). Contre la bienveillance. Paris : Stock.

Morin, E. (1990). Introduction à la pensée complexe. Paris : Éd. Du Seuil.

Paillé, P. et Mucchielli, A. (2003). L'analyse qualitative en sciences humaines et sociales. Malakoff : Armand Colin, coll. U.

Pastré, P., Mayen, P. et Vergnaud, G. (2006). La didactique professionnelle. Revue française de pédagogie, 54, 145-198.

Prairat, E. (2012). Normes et devoirs professionnels. L'esprit déontologique. Les Sciences de l'éducation - Pour l'Ére nouvelle, 45, 123-142.

Prairat, E. (2012). La morale du professeur. Paris : PUF.

Prairat, E. (2013). Les vertus du maître à I'heure de l'école inclusive. La Nouvelle revue de l'adaptation et de la scolarisation, 61, 175-186.

Prairat, E. (2017). Éduquer avec tact. Paris : ESF Sciences humaines, coll. Pédagogies, Questions vives.

Références sur le Web

Algan, Y. Huillery É. et Prost, C. (2018). Confiance, coopération et autonomie : pour une école du xxi ${ }^{\mathbf{e}}$ siècle. Notes du conseil d'analyse économique, 48, 1-12. En http://www.cae-eco.fr/IMG/pdf/cae-note048.pdf (05/04/2019).

Bandura, A. (1986). The Explanatory and Predictive Scope of Self-Efficacy Theory. Journal of Social and Clinical Psychology. 4, 359-373. https://guilfordjournals.com/doi/pdf/10.1521/jscp.1986.4.3.359 (03/09/2019).

Becker, A. (2015). Situation de handicap : « bienveillance ou « exigence »? Contrepied, 12, 3. http://www. epsetsociete.fr/EPS-Sport-et-handicap (06/08/2019).

Bernardin, J. (2013). Cerner la notion de bienveillance éducative face à des élèves de milieu populaire. Communication présentée aux Assises de l'éducation, St Etienne-du-Rouvray/ Insa de Rouen, France, novembre. http: //www.ozp.fr/spip.php?article19617 (04/08/2019).

Dulot, A., Bonneau, F., Combani M.-F., Forestier, C., Mons, N. (2012). Refondons l'École de la République : le rapport de la concertation. https://www.vie-publique.fr/sites/default/files/rapport/pdf/124000547.pdf (03/04/2019)

Bucheton, D. et Soulé, Y. (2009). Les gestes professionnels et le jeu des postures de l'enseignant dans la classe : un multi-agenda de préoccupations enchâssées. Education \& didactique, 3, 3, 29-48. Doi.org/10.4000/educationdidac tique.543 (11/04/2020). 
Colin J. (2011). Analyse de l'intervention d'un enseignant d'EPS auprès d'handicapés mentaux en classe ULIS et en classe de $\sigma^{e}$. Mémoire de master 2 Recherche, Sport Culture Éducation. Université Rennes 2. https:// apprendreeneps.files.wordpress.com/2013/03/2011-m2-justine-colin1.pdf (06/05/2019).

Dupont, J.-P., Carlier, G., Gérard, P. et Delens, C. (2009). Déterminants et effets de la motivation des élèves en éducation physique : revue de la littérature. Les Cahiers de Recherche en Éducation et Formation, 73, 4-31. https://halshs.archives-ouvertes.fr/halshs-00561544/document (10/06/2019).

Ebersold, S., Plaisance, É. et Zander, C. (2016). École inclusive pour les élèves en situation de handicap. Accessibilité, réussite scolaire et parcours individuels. Cnesco, Conférence de comparaisons internationales. https: //halshs. archives-ouvertes.fr/halshs-01445378/document (09/05/2019).

Fayol, M. (2015). Apprendre le nombre et les opérations. Que savons-nous des difficultés? Communication aux journées de l'Association des professeurs de mathématiques de l'enseignement public, Laon, France, octobre. https: //www.apmep.fr/IMG/pdf/Conf Fayol.pdf (15/04/2020).

Lapeyronnie, D. (2014). Pour une École innovante : synthèse des travaux du Conseil national de l'innovation pour la réussite éducative. https://www.education.gouv.fr/sites/default/files/2020-02/2014-rapport-cnire-Web-366914pdf-31736.pdf (08/04/2019).

Marcel, J.-F. (2018). L'émancipation au risque de la bienveillance. Questions Vives, 29. https://journals.openedition. org/questionsvives/3526 (03/07/2019)

Meirieu, P. Connaître (l'enfant, l'élève). Petit dictionnaire de pédagogie. https://www.meirieu.com/DICTIONNAIRE/ connaitre.htm (15/04/2020).

Perrenoud, P. (1999). Former les enseignants dans des contextes sociaux mouvants : pratique réflexive et implication critique. Communication présentée au congrès de l'Association nationale pour la recherche en éducation, Caxambu, Brésil, septembre. https://www.unige.ch/fapse/SSE/teachers/perrenoud/php_main/php_1999/1999 26.html (04/08/2019)

Reto, G. (2017). La bienveillance à l'École : vers un changement de paradigme? Recherches \& éducations. http: //journals.openedition.org/rechercheseducations/4389 (04/09/2019).

Ribaupierre A. (2015). Pourquoi faut-il étudier la variabilité intra-individuelle lorsqu'on s'intéresse au développement cognitif? In J. Juhel et G. Rouxel (dir.). Différences et variabilité en psychologie (p. 159-178). Rennes : Presses universitaires de Rennes. https://pdfs.semanticscholar.org/3792/8f4568884ddc1fbacbc3a0b4e5cccaa06b08. $\operatorname{pdf}(15 / 04 / 2020)$.

Roelens, C. (2017). Autorité éducative bienveillante et éthique. Ethique en éducation et en formation, 4, 92-107 https://www.erudit.org/en/journals/ethiqueedufor/2017-n4-ethiqueedufor03626/1045191ar.pdf (12/06/2019).

Saillot, É. (2018). Conforter une école bienveillante et exigeante : représentations, préoccupations et pratiques déclarées ». Questions Vives, 29. http://journals.openedition.org/questionsvives/3280 (08/08/2019).

Sarremejane, P. (2017). Le bien-être à l'école : analyse et épistémologie d'une notion ». Recherches \& éducations. http://journals.openedition.org/rechercheseducations/3530 (04/09/2019).

Zakhartchouk, J.-M. (2012). Quelles exigences? Cahiers pédagogiques, 449. https://www.cahiers-pedagogiques. com/Quelles-exigences (06/06/2019).

Zakhartchouk, J.-M. (2017). Ne pas opposer bienveillance et exigence. Cahiers pédagogiques, Hors Série Numérique, 46, 10-11. https://fr.calameo.com/books/003871645bb1a10638b38 (10/08/2019). 